\title{
Visibility Estimation via Deep Label Distribution Learning
}

\author{
Mofei Song, Xu Han, Xiao Fan Liu, and Qian Li
}

\begin{abstract}
This paper proposes an image-based visibility estimation method with deep label distribution learning. To train an accurate model for visibility estimation, it is important to obtain the precise ground truth for every image. However, the ground-truth visibility is difficult to be labeled due to its high ambiguity. To solve this problem, we associate a label distribution to each image. The label distribution contains all the possible visibilities with their probabilities. To learn from such annotation, we employ a CNN-RNN model for visibility-aware feature extraction and a conditional probability neural network for distribution prediction. Our experiment shows that labeling the image with visibility distribution can not only overcome the inaccurate annotation problem, but also boost the learning performance without the increase of training examples.
\end{abstract}

Index Terms-Deep learning, label distribution learning, visibility estimation.

\section{INTRODUCTION}

M ETEOROLOGICAL visibility is a crucial index for reporting daily air quality, which has an important bearing on environmental protection. Visibility has a wide range of applications such as transport safety, industrial and agricultural production. Traditionally, visibility can be estimated by some specialized equipment, such as a transilluminator or foreword scattering sensor [1]. However, since the equipment is usually expensive and inconvenient, we can place the equipment at only a few weather stations to detect the visibility of some fixed scenes. This cannot satisfy the requirement of multiple monitoring applications. To realize ubiquitous monitoring, we can utilize abounding and budget cameras as an alternative. By analyzing the image taken by these cameras, we can obtain meteorological visibility effectively and efficiently.

The image-based visibility estimation constructs the mapping between the image representation and the visibility value. The early visibility estimation methods usually create some physic probabilistic models by meteorological law. Babari et al. [2], [3] employ a non-linear regression model called

This work was supported by National Natural Science Foundation of China (61906036, 42075139), the Open Research Project of State Key Laboratory of Novel Software Technology (Nanjing University) (KFKT2019B02).(Corresponding author: Mofei Song.)

Mofei Song and Xu Han are with the School of Computer Science and Engineering, and the Key Lab of Computer Network and Information Integration (Ministry of Education), Southeast University, Nanjing 211189, China. (e-mail: \{songmf,220184189\}@ seu.edu.cn).

Xiao Fan Liu is with Web Mining Laboratory, Department of Media and Communication, City University of Hong Kong, Hong Kong SAR, China (email: xf.liu@cityu.edu.hk).

Qian $\mathrm{Li}$ is with College of Meteorology and Oceanography, National University of Defense Technology, Changsha 410073, China. (e-mail: public_liqian@163.com).
Koschmieder's theory, which describes the relationship between the contrast distribution and visibility. The measurement of the image contrast can be improved further by the extinction coefficient [4]. However, the performance of these physics models is affected by weather conditions, illumination, and scene variations. It is extremely difficult to hard-code the enormous variability of these complex factors in general.

To improve the adaptiveness, a more sophisticated way is to extract the visibility prediction model from data by deep learning. Li et al. [5] employ a generalized regression neural network (GRNN) and a pre-trained CNN model for visibility estimation. Palvanov and Cho et al. [6] use three streams of deep integrated convolutional neural networks to extract the visibility-aware feature from spectrally filtered images, FFT-filtered images, and RGB images. Since these methods formulate the visibility estimation as a regression problem, we should label the visibilities of all the training images accurately. However, it is difficult to obtain the precise ground truth of image visibility. The human specification of absolute visibility from a single image is unreliable [7]. The specialized equipment cannot generate accurate visibility labels either due to deployment variations and environmental influences.

To overcome the problem, one way is to use image pairs labeled by ranking their visibilities as the supervision [8]. Though it is much easier to achieve the relative visibility annotation given a pair of images, absolute visibility cannot be derived directly from the ranking information. Besides, the annotation burden of relative visibility is increased significantly since there are lots of image pairs to be annotated.

Inspired by a novel machine learning paradigm called Label Distribution Learning (LDL) [9], we propose to label an image by a mixture of visibility values with different intensities, which is described as a distribution. Since such label distribution contains several possible visibility values, the problem of inaccurate annotation is overcome. To obtain such a label, we transform an absolute visibility label provided by humans or equipment into a visibility distribution. The transformation is based on the following observation: the images with close visibility have a high degree of similarity. Accordingly, we adopt one-dimensional Gauss distribution for visibility annotation, where the absolute visibility label is the mean of the distribution. Thus, the absolute visibility has the highest intensity, while the relevant degree of other visibilities is inversely proportional to their distances to the absolute label.

Compared with previous labeling types, the label distribution has two superiorities for visibility estimation. On the one hand, the label distribution gives consideration to both the ambiguity of the visibility label and the convenience 


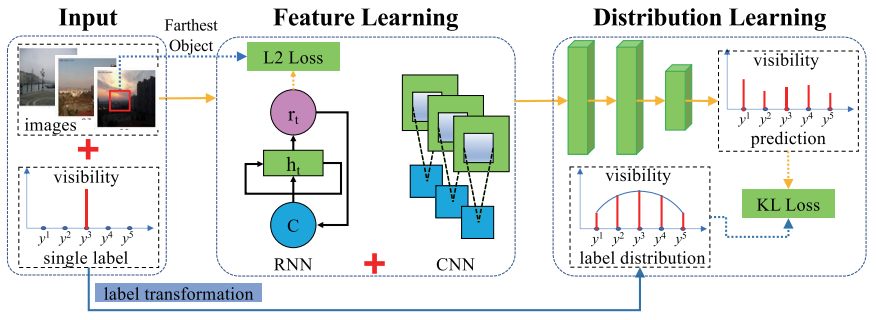

Fig. 1. The overall process of our method. Given the training images and their ground-truth visibilities, we first generate the label distribution by label transformation, and then integrate deep learning and label distribution learning into a unified framework for visibility estimation.

of the visibility prediction. The label distribution improves the robustness of model learning with the uncertain label. Meanwhile, absolute visibility can be obtained directly by searching the highest intensity from distribution. On the other hand, the label distribution can provide more informative supervision. Since every training image labeled by particular visibility is relative to the other closer visibility, it can affect the learned model of adjacent visibilities. In other words, the training images for particular visibility are substantially increased without extra image collection and annotation.

In this letter, a novel visibility estimation method using deep LDL is proposed. Given a set of images labeled by absolute visibility, we first transform the absolute label into a label distribution. Then, we integrate LDL, CNN, and RNN into a unified framework for visibility model learning. The learned model can generate the distribution of all the possible visibilities given a test image. We finally use the visibility with the highest probability as the predicted visibility. The contributions of this study are summarized as follows: 1) We propose to use the label distribution as the supervision for visibility estimation. 2) We design a simple yet effective label transformation method that turns the absolute visibility to the label distribution. 3) We perform some experiments to show that our method can achieve better performance than state-ofthe-art methods.

\section{METHOD}

\section{A. Overview}

We propose a visibility estimation method based on deep LDL. The overall idea is that we label the image visibility with a distribution vector, and integrate deep learning and LDL for visibility estimation. Figure 1 illustrates the whole pipeline of our method. The input is a training image set $S$, and every image $x \in S$ is labeled by an absolute visibility $y$. We also annotate the farthest region of every image by the coordinates of the bounding box $b$. The output is a visibility estimation model, which includes a deep CNN-RNN module and an LDL module. Overall, there are three parts: label transformation, feature learning, and distribution learning. In the following, we describe each part in detail.

\section{B. Label Transformation}

The goal of label transformation is to generate a label distribution for every training image $x$. Given an image $x$, let the vector $D=\left\{d_{x}^{y_{1}}, d_{x}^{y_{2}}, \ldots, d_{x}^{y_{c}}\right\}$ denote its label distribution, where, $Y=\left\{y_{1}, y_{2}, \ldots, y_{c}\right\}$ is the label space, $c$ is the size of the label space, $d_{x}^{y_{i}} \in[0,1]$ is called description degree of the image $x$, which means the probability of its visibility being $y_{i}$. Due to the complexity of the label distribution, fully manual annotation is impractical. Thus, we prefer an automatic way to generate the distribution from an absolute visibility label $y$.

For visibility estimation, the label space is intrinsically continuous. To ease the learning, we quantize the continuous label space into a discrete space $Y$ with equal step size $\triangle y$. In our method, we set the label space $Y=[1 \mathrm{~km}: \triangle y: 12 \mathrm{~km}]$ (MATLAB notation) and the step size $\triangle y=0.1 \mathrm{~km}$. Thus, the label distribution $D$ of every image $x$ is a $111 \mathrm{D}$ vector, which satisfies $\sum_{i} d_{x}^{y_{i}}=1$.

Given an image $x$ labeled by an absolute visibility $y$, it is reasonable to make the corresponding description degree $d_{x}^{y} \in D$ highest in the final distribution $D$. Meanwhile, since the neighbor visibility looks similar in appearance, we can increase the description degrees which are adjacent to the label $y$. Naturally, the description degree $d_{x}^{y_{i}}$ should be gradually reduced when the visibility $y_{i}$ is far away from the label $y$. Based on this observation, we choose the probability density function of one-dimensional Gauss distribution for label transformation:

$$
d_{x}^{y_{i}}=\frac{1}{\sqrt{2 \pi} \sigma} \exp \left(-\frac{\left(y_{i}-y\right)^{2}}{2 \sigma^{2}}\right)
$$

where, $\sigma$ is a hyper-parameter, which is optimized by a simple grid searching described in Section III.

According to equation 1, we transform the absolute label $y$ into a probability vector. To satisfy $\sum_{i} d_{x}^{y_{i}}=1$, this vector is numerically normalized to generate the expected label distribution $D$. The distribution $D$ is used for the following learning process, which makes the distribution $D^{\prime}$ derived from the deep network consistent with the distribution $D$.

\section{Feature Learning}

To simulate the procedure of humans judging the visibility, we follow the relative CNN-RNN method [8]. Since the method uses a ranked image pair to train the deep network, its network architecture contains two similar CNN-RNN branches. Instead, our method uses only one CNN-RNN model to extract the visibility-aware feature, which is more efficient.

Specially, the CNN-RNN model imitates the coarse-to-fine way to detect the farthest target from the image. The CNN module learns the overall visibility from the global image, while the RNN model simulates the region search to realize the coarse-to-fine attention shift. By combining the CNN and RNN model, the final global feature can be more sensitive to the farthest region in the image, which is an important cue for visibility estimation.

Figure 2 illustrates the architecture of our method. For the CNN module, we follow the design of AlexNet [10] for global feature extraction. Our CNN module contains 7 layers, which includes 5 convolution layers and 2 fully connected layers.

For the RNN module, we construct $K$ layers for each of the first six CNN layers, and 1 layer for the last CNN layer. Totally, there are $6 K+1$ states as a sequential manner in the 


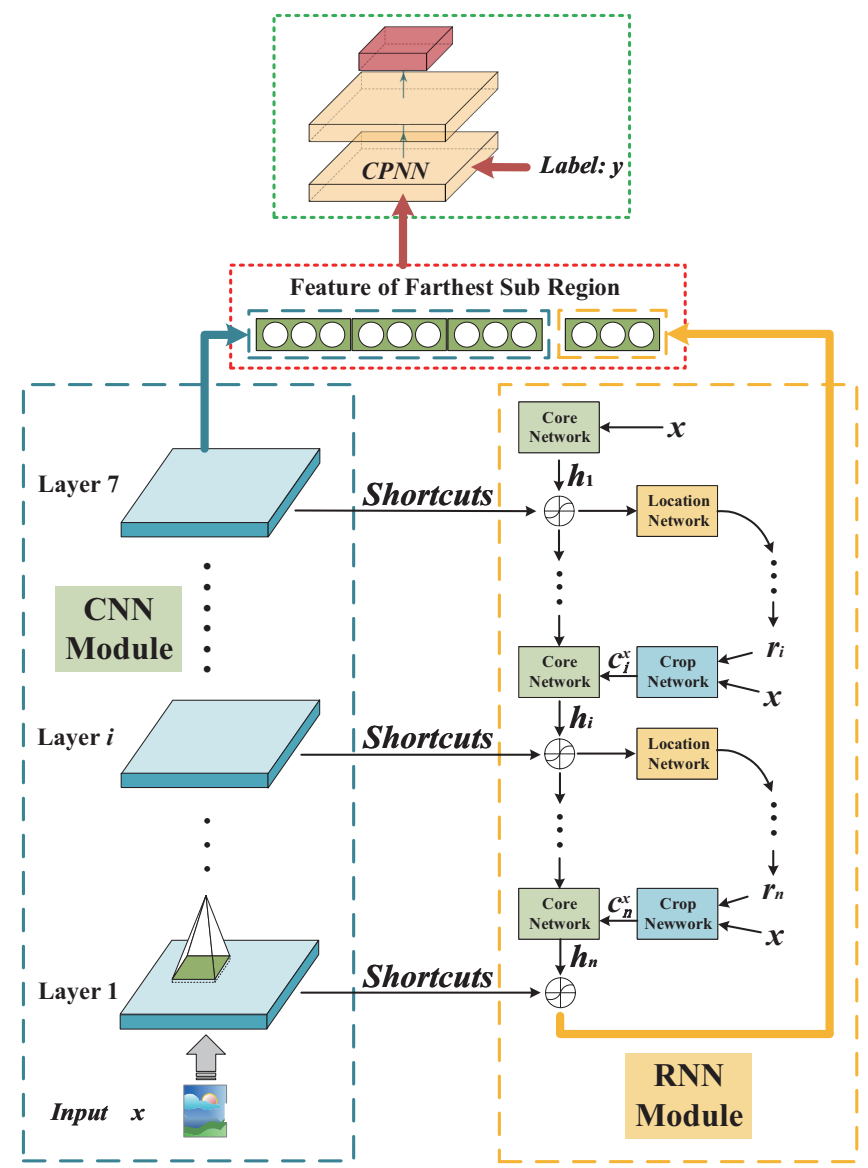

Fig. 2. Our architecture contains three modules: CNN module, RNN module, and $\mathrm{CPNN}$ layer.

RNN module. Every state predicts a bounding box $r_{t}$, and the list of the bounding boxes shows the searching process of the farthest region, namely, from the whole image to the farthest region.

At each recursive step, the $\mathrm{RNN}$ module first crops the image $x$ into a sub-region $c_{t}^{x}$ based on the predicted bounding box $r_{t-1}$, which is the location result of the previous state. Then, the internal state $h_{t}$ is updated by the core network according to the sub-region $c_{t}^{x}$ and the historical state $h_{t-1}$. The generated state $h_{t}$ encodes the knowledge of searching the farthest region. Finally, we use the internal state $h_{t}$ to predict the next bounding box $r_{t}$ by the location network. To exchange the information between $\mathrm{CNN}$ and $\mathrm{RNN}$, some shortcuts connections are added between the $(7-i)$ th $(i=0, \ldots, 6)$ layer of CNN and the $(K i+1)$ th state of RNN.

To train the RNN module, we need to indicate the groundtruth bounding box of every state. To this end, we assume that the list of the bounding boxes is evenly distributed. Accordingly, given the annotated bounding box $b$ of the farthest region, we generate the whole ground-truth list of the bounding boxes $B=\left\{b_{1}, b_{2}, \ldots, b_{6 K+1}\right\}$ by average sampling. During the training phase, we expect to minimize the divergence between the predicted bounding boxes $\left\{r_{1}, r_{2}, \ldots, r_{6 K+1}\right\}$ and the ground truth. Accordingly, we define the objective function of $\mathrm{RNN}$ as a location $L 2$ norm loss:

$$
L_{l}=\sum_{t=1}^{6 K+1}\left\|b_{t}-r_{t}\right\|^{2}
$$

To integrate the information of $\mathrm{CNN}$ and $\mathrm{RNN}$, the output of the CNN module and the last state $h_{6 K+1}$ of the RNN module are concatenated into a global vector representation $f$ for visibility estimation.

\section{Distribution Learning}

To utilize the label distribution $D$, we integrate it into the network architecture. Naturally, we can use several fully connected layers with a softmax layer to turn the feature $f$ into a distribution directly. However, this yields lots of weights between the feature layer and the output layer, which makes it difficult to obtain the optimal solution. Thus, we follow the design of the conditional probability neural network (CPNN) [11].

As shown in Figure 2, CPNN contains three fully connected layers. The input of CPNN contains the feature $f$ and a discrete visibility label $y$. The output of CPNN is a single value $p(y \mid f)$ as the conditional probability. To realize the training, the Kullback-Leibler divergence is employed to measure the difference between the estimated distribution and the groundtruth distribution $D$ :

$$
L_{d}=\sum_{j} d_{x}^{y_{j}} \ln \frac{d_{x}^{y_{j}}}{p\left(y_{i} \mid f\right)}
$$

Finally, we define the entire objective function as the sum of the location loss and the distribution loss:

$$
L=L_{l}+L_{d}
$$

Given the entire objective function, we simultaneously optimize the CNN-RNN module and the CPNN module through back-propagation with stochastic gradient descent [12]. To accelerate the training, we use the $\mathrm{CNN}$ pre-trained by ImageNet. According to the learned model, the predicted visibility could be the one with the maximum description degree.

\section{EXPERIMENTS}

\section{A. Experimental Setup}

Tensorflow is used to implement the models and comparative experiments. The model is optimized by Adam optimizer. During training, the batch size is 64 , the learning rate is 0.00001 , and the epoch is 50. All the experiments are conducted on NVIDIA GeForce RTX 2080Ti.

The method is evaluated on 3 image sets: FROSI (Foggy Road Sign Images) [13], FRIDA (Foggy Road Image Dataset) [14] and RMID (Real Multiview Images Dataset). FROSI and FRIDA are two synthetic datasets. RMID is collected by ourselves, which includes 3000 images. The visibility is labeled based on the report of the weather bureau, ranging from $1 \mathrm{~km}$ to $12 \mathrm{~km}$. For each set, $70 \%$ of images are randomly selected for training and the others are the test images. 


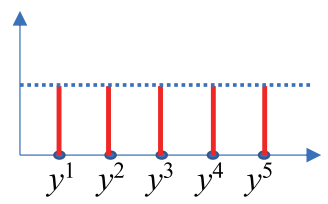

average distribution

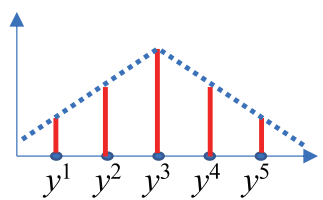

triangle distribution

Fig. 3. The different label distributions: average distribution and triangle distribution

To compare the performance of different methods, we use the mean absolute error (MAE) as the evaluation index, which is defined as:

$$
M A A E=\frac{1}{n} \sum_{i=1}^{n} \frac{\left|y_{i}-\hat{y}_{i}\right|}{y_{i}}
$$

where, $y_{i}$ and $\hat{y}_{i}$ are the ground truth and the predicted visibility of the $i$ th testing image respectively, $n$ is the number of the testing images.

\section{B. Distribution Analysis}

We evaluate our choice of Gauss distribution and the parameter of the standard deviation $\sigma$. To study the influence of the distribution type, we compare the performance of Gauss distribution, average distribution, and triangle distribution. Figure 3 shows the shapes of the other two distributions. To search the optimal standard deviation $\sigma$, we run our method with 5 different values. As shown in Table I, different distributions have a different impact on the prediction results. From the table, we can see that the optimal distribution is the Gauss distribution. Moreover, the value of the standard deviation has a significant effect on the final performance. If the value is too large, the Gauss distribution is very similar to the average distribution. If the value is too small, the Gauss distribution is over concentrated, which degenerates into absolute label. Accordingly, we unify $\sigma^{2}=1.5$ in the following experiment since it leads to the best performance.

TABLE I

RESUlts of DiFFERENT LABEl Distribution.

\begin{tabular}{|c|c|c|}
\hline Distribution & FROSI & RMID \\
\hline Average Distribution & $14.5 \%$ & $19.3 \%$ \\
\hline Triangle Distribution & $15.0 \%$ & $17.9 \%$ \\
\hline Gauss Distribution $\left(\sigma^{2}=2.5\right)$ & $14.2 \%$ & $17.2 \%$ \\
\hline Gauss Distribution $\left(\sigma^{2}=2.0\right)$ & $13.6 \%$ & $16.8 \%$ \\
\hline Gauss Distribution $\left(\sigma^{2}=1.5\right)$ & $\mathbf{1 2 . 1 \%}$ & $\mathbf{1 5 . 8 \%}$ \\
\hline Gauss Distribution $\left(\sigma^{2}=1.0\right)$ & $13.3 \%$ & $16.1 \%$ \\
\hline Gauss Distribution $\left(\sigma^{2}=0.5\right)$ & $15.7 \%$ & $20.4 \%$ \\
\hline
\end{tabular}

\section{CNN-RNN Analysis}

We then explore the influence of the hyper-parameter $K$ in the RNN module. This parameter controls the information exchange between CNN and RNN. To select the optimal value, we run our methods on the set FROSI with three different values. The results are shown in Table II. From the table, we can see that the effect of this parameter is relatively stable. In the following experiments, we set the parameter $K$ as 3 .

TABLE II

RESUlts OF DifFERENT PARAMETER $K$.

\begin{tabular}{|c|c|c|}
\hline$K=2$ & $K=3$ & $K=4$ \\
\hline $16.7 \%$ & $\mathbf{1 2 . 1 \%}$ & $15.1 \%$ \\
\hline
\end{tabular}

\section{Comparison with State-of-the-art Methods}

Table III reports the comparisons of our method and previous state-of-the-art methods on three datasets. According to the results in Table III, our method achieves the best results. By analyzing the estimation results, we can be seen that label distribution is more effective than the absolute label or the ranking label.

TABLE III

COMPARISONS WITH STATE-OF-THE-ARTS AND ABLATION STUDY

\begin{tabular}{|c|c|c|c|c|}
\hline Methods & Label Type & FROSI & FRIDA & RMID \\
\hline VGG16 [15] & absolute & $33.0 \%$ & $26.4 \%$ & $39.8 \%$ \\
ResNet [16] & absolute & $26.3 \%$ & $25.7 \%$ & $30.2 \%$ \\
CNN+GRNN [5] & absolute & $23.8 \%$ & $29.1 \%$ & $35.6 \%$ \\
DHCNN [17] & absolute & $28.6 \%$ & $29.2 \%$ & $41.9 \%$ \\
VisNet [6] & absolute & $21.4 \%$ & $22.9 \%$ & $27.5 \%$ \\
Relative CNN-RNN [8] & ranking & $14.5 \%$ & $13.8 \%$ & $18.3 \%$ \\
\hline Ours (without CNN) & distribution & $27.1 \%$ & $36.9 \%$ & $40.5 \%$ \\
Ours (without RNN) & distribution & $16.8 \%$ & $17.9 \%$ & $20.0 \%$ \\
Ours (without LDL) & absolute & $15.7 \%$ & $15.6 \%$ & $17.4 \%$ \\
Ours & distribution & $\mathbf{1 2 . 1 \%}$ & $\mathbf{1 3 . 7 \%}$ & $\mathbf{1 5 . 8 \%}$ \\
\hline
\end{tabular}

\section{E. Ablation Study}

To prove the effectiveness of different designs, we compare our method with all components and alternatives with one of our choices disabled. We run the following variants:

No CNN - We remove the CNN module from the network.

No RNN - We remove the RNN module from the network.

No LDL - We use the absolute label instead of the distribution and turn it into a regression problem.

We run our method and the variants on the three image sets. As shown in Table III, we can see that our method achieves significant performance boost compared with other variants. Among all the choices, the $\mathrm{CNN}$ module plays the most important role.

\section{CONCLUSION}

We observe that image-based visibility estimation cannot successfully learn precise models when the labels are ambiguous. To solve this problem, we propose a deep label distribution learning framework for visibility estimation. In our method, the visibility of every image is annotated by a label distribution. To learn from such annotation, we integrate CNN, RNN, and CPNN into a unified framework, which locates the farthest region in the image and minimizes the difference between the predicted distribution and the groundtruth distribution simultaneously. The experiment shows that compared with the absolute label or ranking label, label distribution can achieve the best performance for visibility estimation. 


\section{REFERENCES}

[1] M. C. W. van Rossum and T. M. Nieuwenhuizen, "Multiple scattering of classical waves: microscopy, mesoscopy, and diffusion," Rev. Mod. Phys., vol. 71, pp. 313-371, 1999.

[2] R. Babari, N. Hautière, Éric Dumont, R. Brémond, and N. Paparoditis, "A model-driven approach to estimate atmospheric visibility with ordinary cameras," Atmos. Environ., vol. 45, no. 30, pp. 5316-5324, 2011.

[3] R. Babari, N. Hautière, E. Dumont, J.-P. Papelard, and N. Paparoditis, "Computer vision for the remote sensing of atmospheric visibility," in Proc. IEEE Int. Conf. Comput. Vis. Workshops, 2011, pp. 219-226.

[4] Q. Li, Y. Li, and B. Xie, "Single image-based scene visibility estimation," IEEE Access, vol. 7, pp. 24 430-24 439, 2019.

[5] S. Li, H. Fu, and W. Lo, "Meteorological visibility evaluation on webcam weather image using deep learning features," Int. J. Comput. Theory. Eng., vol. 9, pp. 455-461, 2017.

[6] A. Palvanov and Y. Cho, "Visnet: Deep convolutional neural networks for forecasting atmospheric visibility," Sensors, vol. 19, no. 6, p. 1343, 2019.

[7] Koenderink and J. J., "Pictorial relief," Phil. Trans. R. Soc. A., vol. 356, no. 1740, pp. 6-6, 1998.

[8] Y. You, C. Lu, W. Wang, and C.-K. Tang, "Relative cnn-rnn: Learning relative atmospheric visibility from images," IEEE Trans. Image Process., vol. 28, no. 1, pp. 45-55, 2019.

[9] X. Geng, "Label distribution learning," IEEE Trans. Knowl. Data Eng., vol. 28, no. 7, pp. 1734-1748, 2016.

[10] A. Krizhevsky, I. Sutskever, and G. E. Hinton, "Imagenet classification with deep convolutional neural networks," in Proc. Adv. Neural Inf. Process. Syst., vol. 25, 2012, pp. 1097-1105.

[11] X. Geng, C. Yin, and Z.-H. Zhou, "Facial age estimation by learning from label distributions," IEEE Trans. Pattern Anal. Mach. Intell., vol. 35, no. 10, pp. 2401-2412, 2013.

[12] R. Johnson and T. Zhang, "Accelerating stochastic gradient descent using predictive variance reduction," in Proc. Adv. Neural Inf. Process. Syst., vol. 26, 2013, p. 315-323.

[13] J.-P. Tarel, N. Hautière, A. Cord, D. Gruyer, and H. Halmaoui, "Improved visibility of road scene images under heterogeneous fog," in Proc. IEEE Intell. Veh. Symp, 2010, pp. 478-485.

[14] R. Belaroussi and D. Gruyer, "Impact of reduced visibility from fog on traffic sign detection," in Proc. IEEE Intell. Veh. Symp, 2014, pp. 1302-1306.

[15] K. Simonyan and A. Zisserman, "Very deep convolutional networks for large-scale image recognition," in Proc. Int. Conf. Learn. Represent., 2015.

[16] K. He, X. Zhang, S. Ren, and J. Sun, "Deep residual learning for image recognition," in Proc. IEEE Conf. Comput. Vis. Pattern Recognit., 2016, pp. 770-778.

[17] A. Palvanov and Y. Im Cho, "Dhenn for visibility estimation in foggy weather conditions," in Proc. Joint 10th Int. Conf. Soft Comput. Intell. Syst. (SCIS) 19th Int. Symp. Adv. Intell. Syst. (ISIS), 2018, pp. 240-243. 